\title{
FORUM
}

\author{
Harald Müller
}

\section{Großmächtebeziehungen, Abschreckung und nukleare Abrüstung: Ein Perspektivwechsel}

Die Beziehungen der nuklear bewaffneten Großmächte bleiben wegen ihres hohen Schadenspotenzials ein zentraler Gegenstand der Friedensforschung. Gegenwärtig drohen sich überkommene Formen von Mächterivalität neu zu entfalten. Die nukleare Abrüstung, von US-Präsident Obama zu Beginn seiner Amtszeit als politisches Projekt artikuliert, eignet sich als Instrument der Einleitung und Steuerung einer Transformation des Beziehungssystems der Großmächte, durch die dieses von Konfrontation und Machtkonkurrenz auf sicherheitspolitische Kooperation umgestellt wird. Die einzelnen Schritte sind dabei so zu konzipieren, dass sie jeweils über sich hinausweisen und neue Handlungsspielräume eröffnen. Auch muss der Abrüstungsprozess mit einem weiteren Prozess der politischen Konfliktbearbeitung und Konzertierung verbunden werden. Auf diese Weise lässt sich das in das Abschreckungssystem eingeschriebene Sicherheitsdilemma vermindern und schließlich überwinden. Die Alternative ist ein riskanter mehrseitiger Rüstungswettlauf mit unabsehbarem Verlauf.

Schlagworte: Großmächtebeziehungen, Rüstungskontrolle, nukleare Abrüstung, Abschreckung, Systemtransformation

\section{Einleitung}

Die Beziehungen zwischen den Großmächten galten über Jahrzehnte als die Essenz der Internationalen Beziehungen. ${ }^{1}$ Die letzten dreißig Jahre haben dieses Paradigma durcheinander gewirbelt. Die Welt ist bunter geworden und, in der Diktion von

1 Für hilfreiche Kommentare danke ich meinem Kollegen Marco Fey sowie den Verfassern von insgesamt sechs Gutachten in zwei Runden. Besonderen Dank schulde ich jenem Gutachter, der die Empfehlung aussprach: »Hilfreich wäre ein Verweis auf die Arbeiten Harald Müllers zur Rolle von Großmächten [...]«. Wie das Literaturverzeichnis bezeugt, habe ich von dieser unverhofften und unwiderstehlichen Lizenz zur Selbstzitation reichlich Gebrauch gemacht. 
James Rosenau (1990), turbulenter. Eine Vielzahl von Akteuren bevölkert die Beziehungen jenseits des Nationalstaates: Internationale Organisationen mit Akteursqualitäten, die weit darüber hinaus reichen können, bloß die Summe der politischen Willen der Mitgliedsländer zu sein, Nichtregierungsorganisationen, transnationale Netzwerke, internationale Unternehmen. Die Welt wird dadurch nicht unbedingt besser, denn auch Mafia oder al-Qaida mischen mit, aber sie wird sicherlich komplexer.

All das ist unbestritten. Nicht unbestritten ist das relative Gewicht der verschiedenen Akteure. Von einer emergenten Weltkultur ist die Rede (Boli/George 1999; Meyer 2010), von der kosmopolitischen Demokratie (Archibugi 2008), der wachsenden Bedeutungslosigkeit des Staates (Beck 2004). All diese Ansätze überhöhen fraglos erkennbare Tendenzen realpolitischer Entwicklung idealtypisch, um sie anschließend zum realgeschichtlichen Zentraltrend zu erklären. Das ist unvorsichtig. Man tut den vielen plausiblen Diagnosen einer sich ausdifferenzierenden Weltpolitik keineswegs mit dem Hinweis unrecht, dass die zahlreichen funktionierenden Territorialstaaten, unter denen manche konsolidierter und handlungsfähiger sind als vor zehn oder zwanzig Jahren, nicht nur über unvergleichlich variable Machtressourcen verfügen, sondern auch über Strukturen und Prozesse, um diese Ressourcen zur Wirkung zu bringen. Die Ausdifferenzierung der Weltpolitik macht es ihnen sicher schwerer, effektiv ihre Ziele zu erreichen. Carl von Clausewitz hat am Beispiel des Krieges gezeigt, wie die Interaktion widerstreitender politischer Willen dazu tendiert, einen unberechenbaren Vektor aus dem Zusammenwirken der auf Zielerreichung gerichteten, voneinander unabhängigen, gegenläufigen Handlungen zu produzieren (Clausewitz 1980: Kap. I). Dieser Effekt verstärkt sich natürlich mit der Zunahme der Akteure. Zielerreichung wird durch Macht nicht garantiert; aber eine eher große Wirkung in der Umwelt sehr wohl.

Großmachtpolitik bleibt schon deshalb ein zentraler Untersuchungsgegenstand nicht nur der Internationalen Beziehungen, sondern auch der Friedensforschung, weil ihr inhärentes Schadenspotenzial immens ist. Das beginnt mit der Blockade notwendiger internationaler Regelungen - Stichwort Klimawandel oder Regulation der Finanzmärkte - und kulminiert in den Risiken großer Kriege. Es mag in den Hintergrund der öffentlichen Aufmerksamkeit gerückt sein, sollte sich dem in die Tiefe gehenden Blick wissenschaftlicher Analyse aber nicht entziehen: Das nukleare Damoklesschwert hängt nach wie vor über den Köpfen der Menschheit, und in der arkanen Heimlichkeit der Laboratorien und Verteidigungsplanungen arbeiten die Spezialisten unentwegt daran, es weiter zu schärfen. Die Abrüstungsrhetorik mag lauter geworden sein, konkrete Abrüstungsschritte hingegen unterliegen einer bedenklichen Lähmung. Es besteht die paradoxe Situation, dass die Kernwaffen- 
bestände der beiden nuklearen Supermächte USA und Russland zwar weiterhin schrumpfen, da die Vorgaben des NewSTART-Vertrages (Strategic Arms Reduction Treaty) von 2010 implementiert werden, sodass wir von einem Vierteljahrhundert vertraglich geregelter, bilateraler nuklearer Abrüstung sprechen können; zugleich gehen diese Schritte jedoch einher mit einer technischen Modernisierung und einer neuen Politik der Reservehaltung. Zahlreiche Sprengköpfe werden nicht demontiert, sondern rekonstituierbar gelagert. Die vorhandenen Trägersysteme bieten entsprechende »Leerstellen«, die bei Bedarf schnell aufgefüllt werden könnten. Die Abrüstung ist also nur eine Seite der nuklearen Medaille, deren andere fortgesetzte, massive Investitionen in die Aufrechterhaltung der Abschreckung bilden.

Für die Friedensforschung ist es daher geboten, im Sinne einer informierten Frühwarnung emergente Risiken zu benennen, auch und gerade wenn sie nicht von selbst ins Auge springen, und an entsprechenden Gegenstrategien zu arbeiten. Dieser Imperativ ist umso ernster zu nehmen, als in der Tradition der deutschen Friedensforschung die Auseinandersetzung mit pathologischen Aspekten der die Weltpolitik prägenden Großmachtbeziehungen steht, in der historisch und sachlich obsolete, aber mental, kulturell und institutionell verankerte Orientierungen und dementsprechende Interessen das explosive Potenzial der Großmachtkonkurrenz gefährlich aufladen. Die Kombination von psychologischen oder ideologischen Dispositionen und gesellschaftlichen Formationen als Rüstungsursache ist vor allem von Dieter Senghaas sowie von Ernst-Otto Czempiel thematisiert worden (Senghaas 1970; 1972; Czempiel 1979; 1989). Ihre Befunde gewinnen neue Aktualität, je mehr sich in einer mehrseitigen Mächterivalität Charakteristika des Kalten Krieges nicht mehr in einer bipolaren, sondern in einer von Asymmetrien geprägten multipolaren Konstellation auffinden lassen.

Die hier gewählte Perspektive ist also offen normativ, was das Ziel der Friedensforschung betrifft: Es geht darum, die Risiken, die im Zusammenspiel des Konfliktsystems zwischen den großen Mächten mit ihrem angehäuften, vor allem nuklearen, Gewaltpotenzial liegen, zu verstehen und praktische Optionen zu entwickeln, um diese Risiken einzuhegen. Diese normative Zielsetzung hindert indes nicht daran, Handlungsmöglichkeiten mit nüchtern-analytischem Blick abzuschätzen (Müller 2012 a).

Dieser Aufsatz richtet den Blick auf das Verhältnis von nuklearer Abschreckung und nuklearer Abrüstung im Beziehungsgeflecht der Großmächte, das sich augenblicklich in einer dynamischen Bewegung befindet. Er geht von der Grundannahme aus, dass dieses Verhältnis hohe Risiken birgt, zugleich aber durchaus auch Chancen bietet, die Dynamik in eine für die Welt zuträgliche Richtung zu wenden. Eine solche Wendung kommt nicht von selbst. Die wachsende Differenzierung der Welt- 
politik enthält keine Friedens- und nicht einmal eine Abrüstungsgarantie. Der Frieden wird nur gewahrt und die Abrüstung nur erreicht, wenn relevante Akteure, vor allem die Großmächte selbst, diesen Pfad wählen. Diesen Fokus auf Agency verliert nur aus dem Blick, wer sich der Illusion hingibt, unsichtbare strukturelle Kräfte würden das Geschehen determinieren.

Dieser Aufsatz versucht sich an der Konstruktion einer Agenda für Agency, die die nukleare Abrüstung als ein zentrales Element in eine Strategie für die Veränderung der Großmächtebeziehungen integriert. Er verfolgt also weder eine erklärende noch eine verstehende Absicht, sondern stellt einen Versuch dar, einen theoretisch und empirisch begründeten Rahmen für politisches Handeln in der Zukunft zu skizzieren, mit der praktischen Absicht, Überlegungen zu einer Transformation der internationalen Beziehungen beizutragen. Er ist theoretisch informiert durch die von Alexander Wendt (1987) und David Dessler (1989) vor einer Generation im Anschluss an Anthony Giddens' (1984) Strukturierungstheorie herausgearbeitete Beziehung zwischen Struktur und Akteur, in welcher die Struktur beschränkend und ermöglichend, aber nicht determinierend auf das Handeln der Akteure einwirkt. Ideelle Faktoren sind wie materielle Teil der Struktur, als solche aber auch Ressource für die Handlungen der Akteure. Vom Wendt'schen Ansatz, der nach wie vor der am detailliertesten ausgearbeitete im Reich des Konstruktivismus in den IB ist (Wendt 1999), weicht der hier vertretene dreifach ab: Er misst den innerpolitischen/gesellschaftlichen Strukturen und Prozessen gleiches Gewicht in ihrem Wirken auf das auswärtige Handeln zu wie jenen des internationalen Systems; er anerkennt die Rolle von Emotionen im politischen Handeln im Anschluss an Jonathan Mercer $(2010$; 2013) und Richard Ned Lebow (2008), und er räumt - wiederum mit Lebow - dem Moment der Kontingenz einen gewichtigen Einfluss auf den Ablauf der Geschichte ein. Damit ergibt sich ein eklektisches Theoriegebäude (vgl. Sil/ Katzenstein 2011) und eine Indeterminiertheit, die für die positivistische Wissenschaftsauffassung sicher inkorrekt, aus meiner Sicht aber der Sache angemessen ist.

Daher weicht dieser Aufsatz auch im Aufbau von dem Standard-Modell eines Aufsatzes ab, der von Fragestellung über Stand der Forschung, ${ }^{2}$ Erläuterung der Methode, Analyse der zu erklärenden oder verstehenden Empirie zur Auseinandersetzung mit alternativen Erklärungsversuchen und zur Zusammenfassung der Befunde voranschreitet. Er ist praktisch motiviert und zukunftsgerichtet. Ich

2 Mehrere meiner Gutachter haben einen »Forschungsstand « angemahnt, aber keine Hinweise außer denen auf meine eigenen Arbeiten gegeben. Tatsächlich scheint es mir für diese kontrafaktische Exploration keinen Forschungsstand zu geben. Strategieansätze zur nuklearen Abrüstung liegen vor (z. B. Perkovich/Acton 2008; ICNND 2009), aber keiner versucht die Integration mit der Bearbeitung der Großmachtbeziehungen unter Anwendung auf die gegenwärtigen weltpolitischen Prozesse. 
beschreibe im folgenden Kapitel die Großmachtbeziehungen und ihre Risiken (2), umreiße das Konzept der Transformationsstrategie und die Rolle nuklearer Rüstungskontrolle und Abrüstung darin und illustriere es anhand exemplarischer Einzelschritte (3). Von dort aus diskutiere ich das Ziel eines Paradigmenwechsels weg von der nuklearen Abschreckung und setze mich mit der Idee »virtueller Abschreckung « auseinander, welche für manchen prominenten Abrüstungsbefürworter als Endstadium des Abrüstungsprozesses gilt (4). Zusammenfassende Überlegungen schließen den Aufsatz ab (5).

Methodisch betreibt der Aufsatz die Konstruktion eines kontrafaktischen Szenarios (Lebow 2010 a). Kontrafaktisch ist es nicht nur, insofern es sich auf die Zukunft bezieht, sondern auch indem es an zwei »Variablen dreht«: erstens an dem zu beobachtenden Festhalten der Großmächte an ihrem nuklearen Status - der Aufsatz unterstellt Agency in Richtung auf Veränderung; zweitens an dem zu beobachtenden Driften der Großmächte in eine hochriskante Konfliktverflechtung - der Aufsatz unterstellt die Möglichkeit des politischen Willens, der in dieser Verflechtung angelegten Krisenfalle zu entgehen.

\section{Die Problemlage: Risiken der heutigen Großmachtbeziehungen}

Der Titel dieses Aufsatzes enthält eine Paradoxie - seine beiden Teile passen nicht zusammen. Wenn man das nukleare global zero als eine Zukunftsvision versteht, die heute ernsthafte politische Optionen eröffnet und langfristig die Chance auf Realisierung hat, so hat nukleare Abschreckung auf Dauer keine Zukunft. Sie reflektiert ein fundamentales Sicherheitsdilemma zwischen den beteiligten Staaten ebenso wie sie es reproduziert (Booth/Wheeler 2008: 42-61). Das Sicherheitsdilemma entsteht, wenn sich politische Einheiten als Akteure gegenüberstehen, die sowohl zur Kooperation als auch zur Konfrontation bereit sein könnten, ohne dass der eine vom anderen einschätzen kann, für welche Alternative er sich entscheiden wird. Da die eigene Positionierung je nach Einschätzung des Gegenübers drastisch unterschiedlich ist, entsteht eine hoch dilemmatische Situation, da die eigene Entscheidung nicht folgenlos bleibt, sondern auf die Perzeption und daraus entspringende politische Positionierung des Gegenübers einwirkt. Die Bearbeitung des Sicherheitsdilemmas ist daher eine der zentralen Problematiken der internationalen Beziehungen.

Realistische Theorie resigniert vor der Aufgabe, auf diesen circulus vitiosus einzuwirken und beschränkt sich bestenfalls auf Management-Optionen. Der Friedensforschung ist es hingegen immer darum gegangen, über das Sicherheitsdilemma hinaus zu kommen. Dass das möglich ist, hat z. B. die Forschung zu»Sicherheits- 
gemeinschaften« ergeben (Adler/Barnett 1998). Die Idee der vollständigen nuklearen Abrüstung fußt gleichfalls auf der Vorstellung, das Sicherheitsdilemma lasse sich entschärfen und sogar gänzlich überwinden; das kann nur auf Kosten der Abschreckung gehen, die das Dilemma zu kontrollieren versucht, indem sie es perpetuiert.

Wenn man sich hingegen darum sorgt, nukleare Abschreckung zukunftssicher zu machen, dann hat man sich von der Zukunftsvision ohne Sicherheitsdilemma verabschiedet, denn zukunftssicher heißt auch: dauerhaft. Beide Konzepte passen langfristig nicht zusammen und können allenfalls eine kurze Wegstrecke zusammen gehen.

\subsection{Machtverschiebung und Machtübergang}

Der Grund für diese Inkompatibilität liegt also einerseits in den intrinsischen Eigenschaften beider Konzepte, hat indes andererseits auch intensiv mit gegenwärtigen Veränderungen in der Weltpolitik zu tun. Sie sind von einer Machtverschiebung zugunsten aufsteigender Mächte charakterisiert. Chinas und auch Indiens Volkswirtschaften wachsen schneller als die der USA. Wachsende Wirtschaftskraft bedeutet wachsende Macht, ausgreifende Interessen und den Wunsch nach mehr Einfluss (Lemke/Tammen 2006). Und während es stimmt, dass die Position der USA noch stark ist und auf dem Wege der »Aufsteiger « zur linearen Fortsetzung weiterer hoher Wachstumsraten viele Stolpersteine liegen (Beckley 2011), gilt Letzteres ebenso für die weitere wirtschaftliche Entwicklung der USA und des Westens insgesamt. Die Vereinigten Staaten werden durch diese Entwicklung keineswegs machtlos, aber ihre Chancen, aufgrund der hegemonialen Stellung die Welt zu gestalten oder die Einhaltung von Regeln zu erzwingen, die amerikanische Präferenzen reflektieren, sinken beständig (Nye 2002; Lebow 2003: Kap. 8).

Die Machtübergangstheorie hat sich mit solchen Konstellationen eingehend beschäftigt. Sie konstatiert, dass die Standardorientierung der Protagonisten, nämlich der Versuch des jeweiligen Hegemons, den Spitzenplatz zu behaupten, und der Anspruch des jeweiligen »Herausforderers « auf den »Platz an der Sonne«, konflikttreibend sind. Versuche, strategische Vorteile und Statusgewinn in der Auseinandersetzung mit Dritten zu suchen, sowie Rivalitäten zwischen mehreren »Herausforderern« um den »Platz auf dem Sprungbrett« können gleichfalls konfliktverschärfend wirken. Weil all diesen Akteuren auch andere Verhaltenstypen offenstehen, sind systemische Machtverschiebungen indes nicht zwangsläufig konflikt- und kriegstreibend. Die Machtübergangstheorie hat sich überwiegend auf Kriege konzentriert, die durch derartige tektonische Verschiebungen ausgelöst wurden, aber 
die Möglichkeit friedlicher Transitionen eingeräumt. Entscheidend ist, ob es gelingt, einen Zustand (relativer) Zufriedenheit auf beiden Seiten, auf Seiten des (der) »Herausforderer(s)« und des »Hegemons« mit der Installierung der neuen Ordnung zu erreichen. Zufriedenheit hängt von der Perzeption der eigenen Sicherheitsgewährleistung, der Normen und Regeln der neuen Ordnung, der eigenen Partizipationsmöglichkeiten und des eigenen Status ab. Friedliche Machtübergänge stellen hohe Anforderungen an die Anpassungsfähigkeit der Mächte, die im Zuge der Veränderungen relative Einbußen zu verzeichnen haben, und an die Lernkapazität der Aufsteiger, die sich damit vertraut machen müssen, wachsende Verantwortung mit Augenmaß und Selbstbeschränkung auszuüben (zu dieser Problematik ausführlich Rauch 2014). Die Erfüllung beider Anforderungen sind keineswegs Selbstverständlichkeiten: Machtverschiebungen sind historisch mit Kriegsrisiken behaftet. $\mathrm{Ob}$ diese Risiken bewältigt werden oder ob sie die Akteure überwältigen, ist keinen Naturgesetzen unterworfen, sondern ergibt sich aus der Praxis der Schlüsselakteure (Tammen et al. 2000; kritisch Lebow 2010 b: Kap. 4).

\subsection{Akute Beziehungskonflikte zwischen den Großmächten}

Deren Beziehungen sind akut Management-bedürftig. Die Welt lebt seit zwei Generationen ohne Großmächtekrieg und das kriegerische Geschehen hat insgesamt statistisch nachgelassen (Pinker 2011; Lebow 2010 b: Kap. 7). Zu verkennen, dass das auch das Ergebnis harter (diplomatischer) Arbeit und von gutem Glück gewesen ist, wäre sträflich. Ebenso sträflich wäre es, diese erfreuliche jüngere Vergangenheit ohne Weiteres als vermeintliche Gewissheit in die Zukunft zu extrapolieren (Mueller 1989). Man darf nie vergessen, dass Norman Angell aus den ökonomischen und Jean de Bloch aus den militärischen Gegebenheiten am Vorabend des Ersten Weltkrieges - korrekterweise - ableiteten, ein Krieg in Europa sei wider die rationalen Interessen aller großen Mächte (Angell 1910; Bloch [1902] 1972). Sie hatten mit dieser Einschätzung völlig recht, der Krieg kam nichtsdestoweniger.

Wie damals erscheinen auch heute Warnzeichen am Horizont, dass das Vernünftige und das politisch Wirkende nicht zwangsläufig deckungsgleich sind. Schon liest man bei Autoren wie Robert Kagan (2008), Robert Pfaltzgraff (2009), Thérèse Delpech (2012: Kap. 6) oder in der jüngsten nationalen Sicherheitsstrategie Russlands (Sicherheitsrat der Russischen Föderation 2009) von wechselseitigen Feindbildern, die an den Kalten Krieg erinnern. In China unterstellt die politische Elite den Vereinigten Staaten nahezu einhellig eine gegen China gerichtete Eindämmungspolitik (Yao 2010). Indien vermutet im Ausbau der chinesischen Marine und dem erfolgreichen chinesischen Bemühen, im Indischen Ozean eine »Perlenkette« von 
Ankerstationen und Marinestützpunkten zu errichten, eine Umzingelungsstrategie (Mohan 2003; Rafii 2009).

Die Mechanismen gleichen sich: Die Verkennung der eigenen Rolle im weltpolitischen Geschehen bzw. dessen Wirkung auf die Wahrnehmung der übrigen Beteiligten; die Zuschreibung langfristiger strategischer Kontrollfähigkeit und böser Absichten an den vermeintlichen Gegner; die Überschätzung der Machtressourcen anderer Akteure; die Vermutung, der Worst Case sei die reale Zukunft, wenn nicht eigene militärische Maßnahmen verstärkt würden. Die genannten Schriften lesen sich wie Exerzitien der »Pathologien des Rüstungswettlaufs« (Senghaas 1970), deren individual- und sozialpsychologische Grundlagen Robert Jervis in den siebziger Jahren umfassend dargelegt hat (Jervis 1976).

Die Großmächterivalität ist für Teile der Sicherheitseliten zurück auf der Tagesordnung - wenn sie denn je verschwunden war. Die irritierende Dynamik in den Machtverhältnissen wird zum Anlass genommen, scharf konturierte Bedrohungsbilder zu zeichnen und einschlägige Gegenmaßnahmen zu verlangen. Rüstungsdynamiken sind damit sowohl Produkt des politischen Wettbewerbs als auch - als Angelpunkt des Sicherheitsdilemmas - dessen Triebkraft; daraus ergibt sich die Bedeutung von Rüstungskontrolle und Abrüstung als Teil einer Managementstrategie für die Großmachtbeziehungen (dazu Müller/Neuneck 1992). Neu ist, dass der Wettbewerb der »Großen« nicht zwei Staaten umfasst wie im Kalten Krieg, sondern zumindest vier (auch wenn zumeist nur von dreien die Rede ist): Neben den USA, China und Russland muss Indien mit auf die Rechnung gesetzt werden. Der südasiatische Gigant trabt ein Stück hinter China auf der Straße rapider Entwicklung, und ein Stück hinter China entfaltet sich auch in Delhi der alte Großmachtanspruch (Müller 2006: 259-294). Diese vier Akteure heben sich von anderen Mächten ab: Sie sind nuklear bewaffnet und rangieren auf dem komplexen Machtindex des Correlates of War Project auf den Plätzen 1, 2, 3 und 5; zwischen Indien und Russland schiebt sich Japan, das Teil des Konfliktnetzwerks ist, aber als nicht nuklear bewaffneter Staat hier ausgeblendet bleibt. ${ }^{3}$

3 Vgl. hierzu http://correlatesofwar.org/COW2\%20Data/Capabilities/NMC_v 4_0.csv (letzter Zugriff: 23.02.2014). Auch der Wiedereintritt Japans in das Rivalitätsgeschehen ist nicht auszuschlieBen. Die Furcht vor China wächst, das Gefühl der Statusdeprivation auf der Rechten wird stärker, das früher isolierte Denken in nationalen und Status-Kategorien entwickelt sich zum politischen Mainstream (Takahashi 2012). 


\subsection{Der nukleare Faktor}

Die Beziehungen zwischen diesen vier Akteuren sind komplex und konflikthaltig. Am heikelsten sind die Beziehungen zwischen den USA und China (Foot/Walter 2012). Hier hat der Regierungswechsel 2009 in Washington keine Besserung gebracht, wie die ostentative Verstärkung der militärischen Präsenz Amerikas in der pazifischen Region und die heftigen chinesischen Reaktionen dokumentieren. Die Vereinigten Staaten fungieren als vertragsgebundene oder informelle Schutzmacht von Ländern, mit denen China territoriale Konflikte aufweist - Japan, Südkorea, Philippinen, Vietnam - sowie von Taiwan, das China als Teil des eigenen Territoriums reklamiert. Russland empfindet die USA trotz des publikumswirksam zelebrierten »Reset « unter Obama nach wie vor als Bedrohung und als Rivale in Osteuropa, dem Kaukasus sowie in Zentralasien und bildet mit China eine strategische Zweckgemeinschaft, ohne dass diese Beziehung selbst harmonisch wäre - der chinesische Druck auf Ostsibirien ist bis Moskau fühlbar. Indien und China rivalisieren nicht nur in Südost- und Südasien, sondern streiten sich auch um zwei Grenzterritorien in Himalaya und Karakorum. Die indische Gastfreundschaft gegenüber dem Dalai Lama und die chinesische Partnerschaft mit Pakistan reflektieren die Rivalität und verstärken sie zugleich (Gilboy/Heginbotham 2012). Nur zwischen Russland und Indien sowie Indien und den USA sind die Beziehungen eher problemfrei; die indisch-amerikanischen Beziehungen wirken mittlerweile wie eine Entente im Frühstadium.

Die sicherheitspolitische Problematik resultiert daraus, dass sich diese Beziehungen nicht in Konfliktdyaden zerlegen und jeweils einzeln mit der für bilaterale Beziehungen entwickelten Rezeptur bearbeiten lassen. Sie sind vielmehr durch die genannten Bündnisbeziehungen und die Überlappungen von Interessengebieten ein komplexes und daher eskalationsfähiges Beziehungsgeflecht.

Die vier Großmächte sind alle nuklear bewaffnet und ihre sicherheitspolitischen Beziehungen definieren sich zu einem erheblichen Maße durch diese nukleare Bewaffnung. Das nukleare Verhältnis lässt sich keineswegs nur im Sinne stabiler Abschreckung beschreiben; auch die optimistische Einschätzung, Kernwaffen seien im Wesentlichen »in den Hintergrund « getreten, verkennt deren kontinuierlich zentrale Rolle. Sie ergibt sich aus der stets vorhandenen unterschwelligen Furcht, die Kernwaffen könnten Teil eines Gesamtdispositivs ${ }^{4}$ und einer Grand Strategy sein, die auf hegemoniale Ziele, wenn nicht auf die Demütigung, Marginalisierung oder

4 Der Begriff »Dispositiv« wird synonym mit dem US-amerikanischen militärtechnischen Term posture verwendet. 
Beseitigung des Gegners oder der Gegner, ausgerichtet sind. ${ }^{5}$ Umgekehrt gewinnen die nationalen Arsenale für diejenigen Mächte, die sich als die schwächere Konfliktpartei sehen, die altbekannte Funktion einer existenziellen Sicherheitsgarantie. So sind Russland und China darauf bedacht, ihr Abschreckungsdispositiv gegenüber einer von ihnen wahrgenommenen zweifachen Bedrohung durch die USA abzusichern, nämlich der unterstellten künftigen Fähigkeit, weitreichend und zielgenau mit konventionellen Mitteln zu operieren (Prompt Global Strike) und einen gegnerischen Zweitschlag mit einem gestaffelten, integrierten regionalen/nationalen Raketenabwehrschirm wirksam zu bekämpfen (Fingar 2011; Cimbala 2013). Welche Rolle im Weltraum stationierte amerikanische Waffen künftig spielen könnten, bleibt unklar, die Phantasie allein erregt in Moskau und Bejing bereits weitere Befürchtungen (Neuneck/Rothkirch 2006). Indien sieht seine Kernwaffen als Existenzsicherung gegenüber dem konventionell schnell aufrüstenden China (Gilboy/ Heginbotham 2012) so wie Pakistan, der weitaus weniger mächtige »Appendix« des genannten Konfliktsystems, sein Arsenal als Lebensversicherung gegen Indien versteht. Die Konfliktkonstellation enthält vor allem für die amerikanisch-chinesische Dyade erhebliche Stabilitätsrisiken (Goldstein 2013).

Konsequenterweise bemüht sich Russland, die Zweitschlagsfähigkeit seines Kernwaffenarsenals bei weiterer quantitativer Schrumpfung durch qualitative Veränderungen abzusichern und hat sich dabei zum Rückgriff auf MIRV (multiple independently targetable re-entry vehicle), fortgeschrittene Täuschkörper und anscheinend auch auf Endphasenmanövrierfähigkeit der neuen Raketengeneration konzentriert. Russland hält außerdem eine vierstellige Zahl nichtstrategischer Kernwaffen vor, um die konventionelle Überlegenheit der NATO zu kompensieren (spiegelbildlich zur NATO-Strategie während des Kalten Krieges), aber auch, um befürchtete konventionelle Ungleichgewichte im Vergleich zu China im Fernen Osten auszugleichen.

China baut seine nuklearen Bestände zwar mit erstaunlich »ruhiger Hand«, aber kontinuierlich aus und verbindet quantitativen Aufwuchs mit qualitativen Verbesserungen (Mobilität zu Lande und zur See, Reichweitensteigerung und anscheinend gleichfalls »VerMIRVung«). Zugleich entwickelt es Fähigkeiten gegen Satelliten, um im Konfliktfall die USA an einer neuralgischen Front bedrohen zu können (Lewis 2007). Amerika beschuldigt China und auch Russland einer exzessiven Entwicklung offensiver elektronischer Kriegsführung, unternimmt aber selbst große Anstrengungen in diesem Sektor. Indien orientiert sich im nuklearen Rüstungs-

\section{Zum Folgenden siehe im Überblick Kile et al. (2012).}


geschehen an China und versucht zwar, Redundanzen zu vermeiden, steuert aber auf eine komplette nukleare Triade zu. ${ }^{6}$

Von dieser Warte aus betrachtet, ist die Zielsetzung, die George Shultz, William Perry, Henry Kissinger und Sam Nunn vor nunmehr fast sechs Jahren im Wall Street Journal formuliert haben (Shultz et al. 2007), nämlich die nuklearwaffenfreie Welt, nicht nur als präventive Behandlung des Problems von nuklearem Terrorismus und ausufernder Proliferation zu sehen und schon gar nicht als Formulierung eines utopischen Menschheitstraums. Für das Management der Großmachtbeziehungen ist nukleare Abrüstung vielmehr vor allem ein notwendiges, wenn auch nicht hinreichendes Instrument einer Transformation ihrer Beziehungen, um Vertrauen zwischen ihnen zu schaffen, das Risiko einer unkontrollierbaren Eskalation radikal abzusenken und intensive sicherheitspolitische Kooperation zu etablieren. So würde auch die Behandlung anderer Fragen internationaler Governance, von der regionalen Sicherheit über die Proliferation bis hin zu Energie- und Handelsfragen, erleichtert. All diese Fragen, bei denen es auch um die Verteilung von Kosten und Nutzen zwischen den Großmächten geht, lassen sich nämlich kaum konstruktiv in Angriff nehmen, wenn die Großmachtbeziehungen von Feindseligkeit charakterisiert sind. Das Problem der »relativen Gewinne« (Grieco 1990) ist zwar keine naturgegebene Konstante internationaler Beziehungen, aber eine dominierende Orientierung rivalisierender Großmächte, die sich in einer »hobbesianischen« Welt gefangen wähnen.

\section{3. Überlegungen zu einer Transformationsstrategie}

Praxeologische Überlegungen, d. h. die Entwicklung von politischen Handlungsoptionen, werden oft unabhängig von theoretischer Reflexion und ohne einen konzeptionellen Rahmen in Angriff genommen, so als wären Theorie und Praxis tatsächlich unabhängige Bereiche. Viele Wissenschaftler dürften die Erfahrung des Autors teilen, dass die Aufmerksamkeit vieler Praktiker nachlässt, wenn theoretische Überlegungen in die Diskussion einfließen. Andererseits stehen natürlich hinter jeder Praxis - explizit oder implizit - theoretische Überzeugungen. Deshalb sollen die folgenden Reflexionen auf die Praxis in einen konzeptionellen Rahmen eingebettet werden.

6 Pakistan glaubt, Parität mit Indien wahren zu sollen und folgt angesichts der konventionellen indischen Überlegenheit einer Doktrin des Ersteinsatzes. Die südasiatische Fußnote zur Großmächterivalität ist insoweit der brisanteste Teil der globalen Rüstungskonkurrenz. Nordkorea bleibt in einer ostasiatischen Krisenlage ein zusätzlicher Risikofaktor. 


\subsection{Konzeptionelle Voraussetzungen}

Die im Folgenden skizzierte Strategie unterstellt, dass ein »Konfliktmanagement« zwischen den Großmächten möglich ist. Diese Möglichkeit bedeutet keineswegs eine sichere Prognose, dass sie auch genutzt wird. ${ }^{7}$ Der Versuch, die Entwicklung hochkomplexer sozialer Systeme von außen zu steuern, scheitert bekanntlich häufig an einer Kombination verschiedener widriger Umstände.

Erstens durchschauen die von außen steuernden Akteure die Komplexität nicht, wissen daher nicht, wo die Stellschrauben sind und/oder drehen an den falschen, mit der Folge, dass die Effekte nicht in die erwünschten Richtungen gehen. Auch die Sicherheitspolitik der Großmächte weist hohe Komplexität auf, und die Zahl der sich in diesem Feld tummelnden Akteure hat gleichfalls zugenommen (Booth 2005). Richtungsentscheidungen in diesen Beziehungen und in der damit verknüpften Kernwaffenpolitik sind jedoch ebenso immer noch Sache eher kleinerer Gruppen von Entscheidungsträgern.

Zweitens besteht das "Clausewitz-Problem« der Interaktion von Antagonisten, die jeweils die andere Seite überwinden wollen und die so einen unberechenbaren Vektor produzieren. Hier ist nicht Komplexität, sondern der unüberbrückbare Gegensatz der Akteure der entscheidende Faktor. Hiergegen setzen Steuerungsversuche in friedlicher Absicht eine grundlegende Erfolgsbedingung voraus: Empathie für Interessen, Befürchtungen und erwartbare Reaktionen der Partner. Anders als im Krieg ist nicht die Überwindung des Gegners, sondern die der Feindseligkeit das Ziel des steuernden Akteurs. Die inkrementalistische Abrüstungstheorie hat dies im Einzelnen herausgearbeitet (Osgood 1970; Thränert 1986). Auf der Akteursebene ist die mittlerweile beachtlich starke und gut organisierte transnationale Bewegung, die sich die nukleare Abrüstung auf ihre Fahnen geschrieben hat, eine begünstigende Rahmenbedingung. Im Unterschied zu früheren Abrüstungskampagnen verfügt sie nicht nur über mehr Leitorganisationen, sondern reicht auch bis in die Mitte des »Sicherheitsestablishments« hinein.

Transformative Strategien sind keine Utopien jenseits politischer Realität. Wie die historische Erfahrung zeigt, können sie Erfolge vorweisen. Die deutsche Ostpolitik der 1970er Jahre und Michail Gorbatschows Neuansatz in der sowjetischen Außen- und Sicherheitspolitik nach 1985 waren erfolgreiche Initiativen zur Transformation eines Konflikts. Beide stellten den Versuch dar, aus einem System struk-

7 Das scheint das Missverständnis eines meiner Gutachter gewesen zu sein. Ein kontrafaktisches Szenario erhebt nicht den Anspruch auf hohe Eintrittswahrscheinlichkeit - das hieße den Sinn der kontrafaktischen Methode zu verkennen. Es geht vielmehr darum, einen politischen Möglichkeitsraum sowohl bewusst als auch plausibel zu machen (Lebow 2010 a: Kap. 2). 
tureller Feindseligkeit durch eine Verhaltensumstellung auszubrechen, die sich in ersten Schritten auf den Kern des Sicherheitsdilemmas (Booth/Wheeler 2008: 1-18) aus der Perspektive des Gegenübers fokussierte: Die Ostpolitik arbeitete daran, die Furcht der östlichen Nachbarn vor dem deutschen Revanchismus abzubauen und so dem Militarismus des Warschauer Pakts den Anlass zu entziehen (Brandt 1968). Gorbatschows Initiativen zielten auf westliche Besorgnisse hinsichtlich der sowjetischen militärischen Überlegenheit. Beiden ging es um die Überwindung der Feindseligkeit (English 2000), wobei die deutsche Ostpolitik die Änderungen der sowjetischen Außen- und Sicherheitspolitik im folgenden Jahrzehnt möglich machte. ${ }^{8}$

Die Herausforderungen für eine Transformationsstrategie in der heutigen Konstellation erscheinen angesichts erfolgreicher historischer Beispiele überwindbar, sie unterliegen indes keiner automatischen Transformationsmechanik, sondern bedürfen der strategisch intelligenten Agency. ${ }^{9}$ Überwiegend muss diese Agency von den beteiligten Mächten selbst kommen. Auf dieser Grundlage arbeite ich zunächst die Risiken der gegenwärtigen Trends in den Großmächtebeziehungen heraus und identifiziere die Rolle der Kernwaffen darin. Ich argumentiere, dass für die Umgestaltung der Beziehungen das transformatorische Potenzial von Rüstungskontrolle und Abrüstung nutzbar gemacht werden muss. Der Artikel exemplifiziert dann - in Anlehnung an die Erfahrungen in der Schlussphase des Kalten Krieges - anhand ausgewählter Abrüstungsschritte, wie die Akteure solche Maßnahmen strategisch einsetzen und so auf die Großmächtebeziehungen wirken können und wie sie umgekehrt davon profitieren, diese Beziehungen von Rivalität auf Kooperation umzustellen. Ich würdige anschließend die ambivalente Wirkung des Abschreckungsverhältnisses und plädiere anhand einer Kritik des Konzepts der »virtuellen Abschreckung« dafür, das Abschreckungsverhältnis dauerhaft durch Sicherheitskooperation zu ersetzen, um eine Stabilisierung der Großmächtebeziehungen zu erreichen und damit die Grundlage für die vollständige Abschaffung der Kernwaffen zu legen.

Konstruktivisten haben die Transformation von politischen Beziehungssystemen in ihren materiellen, kulturellen und psychologischen Dimensionen wiederholt thematisiert. So hat Alexander Wendt den Übergang von einer hobbesianischen zu einer lockeanischen und schließlich zu einer kantianischen Welt diskutiert (Wendt

8 Einer meiner Gutachter verlangte den Nachweis, dass diese Erfahrung nach nahezu dreißig Jahren auf heutige Verhältnisse übertragbar sei. Diese Forderung überfrachtet die kontrafaktische Methode. Ich stelle als Mindestbedingung ihrer Anwendbarkeit fest, dass keine grundlegenden Umstände erkennbar sind, die dieser Übertragbarkeit im Wege stehen - auch der Gutachter hat keinen solchen Umstand angeführt.

9 Vgl. zu diesem Punkt Lebow (2010 a: Kap. 4). 
1999), Ken Booth und Nick Wheeler haben den Wandel von Anarchie über Gesellschaft zu Gemeinschaft skizziert (Booth/Wheeler 2008: Kap. 11) und Ned Lebow hat die weltweit wachsende Skepsis gegenüber dem Krieg analysiert (Lebow 2010 b: Kap. 7). In diesen Konzeptionen sind die materiellen Verhältnisse nur als Schranken und Opportunitätsstrukturen für das Handeln wichtig, aber nicht determinierend. Mit der Verschiebung der ideationalen Strukturen durch die Praxis der Akteure ändern sich auch entweder die materiellen Verhältnisse oder das Verständnis ihrer Bedeutungen.

Der entscheidende konzeptionelle Schritt für eine Transformationsstrategie der Großmachtbeziehungen unter heutigen Bedingungen ist die Auflösung einer strikten Sequentialisierung zwischen politischen und rüstungskontrollpolitischen Fortschritten. ${ }^{10}$ An ihre Stelle tritt die Rückkoppelung beider, woraus Synergien aus parallelen Änderungen in beiden Feldern resultieren. Das transformatorische Potenzial von Rüstungskontrolle und Abrüstung kann sich nur in enger Verzahnung mit einem kooperationsgerichteten politischen Inkrementalismus entfalten, wirkt dann aber als Katalysator für die Reduzierung des Sicherheitsdilemmas (siehe unten). Damit grenzt sich diese Strategie von den im nuklearen Nichtverbreitungsvertrag (NVV) aufeinanderprallenden Konzepten ab, indem sie deren brauchbare Elemente in einer Synthese verbindet: Während dort die Kernwaffenstaaten nur kleinste Schritte für möglich halten und auf die widrigen »politischen Rahmenbedingungen « verweisen, mit dieser Argumentation die Abrüstung aber in die unabsehbare $\mathrm{Zu}$ kunft verschieben, befürworten die blockfreien Staaten eine »Big-Bang«-Strategie, der zufolge mit einem einzigen Akt - einer Kernwaffenkonvention oder einem rechtsverbindlichen Zeitziel - die Entscheidung für die nuklearwaffenfreie Welt ohne hemmende Rücksicht auf politische Rahmenbedingungen fixiert wird (Müller 2012 b). Beide Strategien lassen zwischen »jetzt« und »dann« eine Leerstelle, die mein Ansatz konzeptionell auszufüllen sucht.

\subsection{Das transformative Potenzial von Rüstungskontrolle und Abrüstung}

Die These vom transformativen Potenzial von Rüstungskontrolle und Abrüstung wirkt auf den ersten Blick kontraintuitiv, stellt doch »Stabilität« das Kernziel von Rüstungskontrolle dar (Müller/Schörnig 2006: 124-125). Die Begriffsanalyse legt

10 Colin Gray (1992) postuliert eine kausale Sequentialisierung: Rüstungskontrolle funktioniere nur unter der Bedingung einer entspannten politischen Beziehung. Sein vermeintlicher empirischer Beleg ist aber zu grobkörnig, um die Wechselwirkung beider Variablen erfassen zu können. Sie reflektiert daher lediglich die in der theoretischen Perspektive Grays a priori angelegte Annahme über deren Beziehung. 
jedoch offen, dass paradoxerweise das nahezu zwangsläufige Resultat gelingender Rüstungskontrolle die (wünschenswerte) Destabilisierung einer im Sicherheitsdilemma eingefrorenen Feindbeziehung ist. Dieses Stabilitäts-/Destabilisierungsparadox konnte nur übersehen werden, weil der amerikanische Mainstream Abschreckung und Rüstungskontrolle überwiegend in einem materiellen, technischen Rahmen fixiert und dabei vernachlässigt, dass es sich in erster Linie um ein soziales Verhältnis mit signifikanten psychologischen Dimensionen handelt.

Das Sicherheitsdilemma, innerhalb dessen Abschreckung und Rüstungskontrolle funktionieren, ist eine Wahrnehmungskonstruktion, die dem Gegenüber eine potenzielle Angriffsabsicht unterstellt und insoweit Gegenmaßnahmen notwendig erscheinen lässt, die identische Wahrnehmungen und Einschätzungen auf der Gegenseite hervorrufen bzw. verstärken (Booth/Wheeler 2008: 1-18). Rüstungskontrolle will diese zu Präemptionsstrategien tendierende Spiralbewegung hemmen, indem ihre Maßnahmen auf den Abbau physischer Angriffspotenziale mit Überraschungsund Entwaffnungseffekten abzielen. Da gelingende Rüstungskontrolle nur durch Einvernehmen zu Stande kommen kann, vereinbarte Maßnahmen also regelhaft den Sicherheitsinteressen beider Seiten entsprechen, signalisieren diese Maßnahmen in signifikanter Weise, dass der Gegner keine Absichten hegt, eine Aggression vorzubereiten. Die Wahrnehmung dieser Signale auf beiden Seiten hat Folgen für die gesamte Struktur des Verhältnisses, das ja auf der konstruierten Möglichkeit eben solcher Absichten beruht. Die vermeintlichen Gewissheiten über die Gegenseite kommen ins Wanken; positivere Hypothesen über die Natur des vermeintlichen Feindes werden möglich. Damit entsteht ein Anreiz, diese Hypothesen durch weitergehende Maßnahmen zu testen. Deren Erfolg dekonstruiert das frühere Feindbild zusehends und eröffnet Chancen weitergehender politischer Annäherung. Erfolgreiche Rüstungskontrolle geht daher in Abrüstung über, wenn nicht Kräfte der Beharrung (z. B. der »militärisch-industrielle Komplex«; vgl. Homolar 2012) den Prozess stoppen und wieder umkehren (wie in den späten 1970er und wieder in den späten 1990er Jahren geschehen).

Das historische Modell, an dem sich diese strategische Verortung der (nuklearen) Rüstungskontrolle und Abrüstung in einem umfassenden Transformationsprozess orientiert, ist die von Gorbatschow eingeleitete Schlussphase des Kalten Krieges, in der keineswegs, wie es der neokonservative Mythos darstellt, Ronald Reagan die Sowjetunion totgerüstet hat. ${ }^{11}$ Ohne die westliche Bereitschaft, auf die Offerten Gorbatschows mit Zusammenarbeit zu reagieren, wäre nicht der Kalte Krieg zu

11 Dazu kritisch: Lebow/Stein (1994). 
Ende gegangen, sondern stattdessen das Gorbatschow-Experiment. ${ }^{12}$ In diesem Wechselspiel von Schritten, das den Konflikt in eine Kooperationsspirale überführte, spielten Rüstungskontrolle und Abrüstung eine Schlüsselrolle (Evangelista 2004).

Es steht zwar außer Frage, dass der Rüstungskontroll- und Abrüstungsprozess dieser Jahre die Konflikttransformation nicht alleine getragen hat - das würde die Rolle der politischen Schritte, von der allmählichen Lockerung des sowjetischen Griffs auf Osteuropa über die gemeinsame Konfliktbeilegung in Lateinamerika, im südlichen Afrika und in Südostasien bis zum Schulterschluss im Sicherheitsrat der Vereinten Nationen, sträflich unterschätzen. Genauso kurzsichtig wäre es indes, den Anteil zu übersehen, den Rüstungskontrolle und Abrüstung am beständigen Aufbau von Vertrauen und am kontinuierlichen Abbau des immensen Sicherheitsdilemmas getragen haben, das zuvor die Ost-West-Beziehungen weitgehend dominiert hatte. Es begann mit der Signalwirkung, die Gorbatschows überraschende Aufhebung von Restriktionen bei der Manöverbeobachtung im Rahmen des Helsinki-Prozesses ausübte (1986), entwickelte sich über den historischen Gipfel in Reykjavik (1986), den INF-Vertrag (Intermediate-Range Nuclear Forces Treaty, 1987), den Aufbaustopp für SDI (Strategic Defense Initiative) durch den amerikanischen Senat (1987), den Rückzug von 500.000 sowjetischen Soldaten aus Osteuropa (1988), und kulminierte schließlich in den Verhandlungen (1989/1990) zu START und dem Vertrag über Konventionelle Streitkräfte in Europa (KSE-Vertrag). In diesem Prozess verwob sich abrüstungspolitischer Fortschritt mit politischen Fortschritten, wie der Aufgabe der Breschnew-Doktrin, der Beteiligung der Sowjetunion an regionaler Konfliktbeilegung sowie dem Abzug aus Afghanistan (1988), zu einem dynamischen Prozess, der im Fall der Mauer, der Demokratisierung Osteuropas und der Charta von Paris endete (Evangelista 2004).

Eine vergleichbare katalytische Rolle sollte dem nuklearen Abrüstungsprozess in einer Entspannungsdynamik im heutigen Großmächtequartett zukommen. Ohne eine solche Rolle dürfte es wohl weder eine Verbesserung der Beziehungen noch nukleare Abrüstung geben, sondern eine Verhärtung der Konfliktfronten und einen unberechenbaren mehrseitigen Rüstungswettlauf.

12 Im Sommer 1991 verbrachte ich eine denkwürdige Stunde am Strand von Almeria mit einem befreundeten hochrangigen sowjetischen Diplomaten. Er erzählte mir, dass im Frühjahr 1989 angesichts der NATO-Pläne, ein Nachfolgesystem für die Kurzstreckenrakete Lance zu beschaffen, in Moskau Putsch-Stimmung bestand. Konservative Kreise sahen die anstehende NATO-Entscheidung (von den USA und Großbritannien befördert, von Deutschland abgelehnt) als Lackmustest für die gesamte Sicherheitspolitik Gorbatschows. Eine Beschaffungsentscheidung der NATO - die im Frühjahr auf unbestimmte Zeit ausgesetzt wurde - hätte vermutlich die Absetzung Gorbatschows bedeutet: kein Fall der Mauer, keine deutsche Einigung usw. 


\subsection{Exemplarische Schritte der Vertrauensbildung in transformativer Absicht}

Die nachfolgenden, exemplarischen Überlegungen setzen stets voraus, dass parallel zu Abrüstungsschritten der Versuch unternommen wird, die politischen Kernprobleme zwischen den Großmächten zu bearbeiten - etwa einen Modus Vivendi für das südchinesische Meer und die Taiwan-Frage zu finden, der Gewaltanwendung ausschließt, ein System der Energiesicherheit zu schaffen, das alle großen Importeure fossiler Treibstoffe umfasst, zum Beispiel die Erweiterung der Internationalen Energie-Agentur (IEA) um China und Indien (Causevic 2012), die Beilegung der indisch-chinesischen Grenzfrage auf der Basis des Status Quo und das Einfrieren der NATO-Erweiterung. Keines dieser Szenarien erscheint unerreichbar: Der Eintritt Chinas und Indiens in die IEA würde die Aussichten erhöhen, größere Angebotskrisen bei fossilen Rohstoffen zu tragbaren Kosten zu bewältigen. Der Status Quo der indisch-chinesischen Grenzen lässt sich nur um den Preis eines größeren Krieges ändern, woran keine der beiden Seiten interessiert ist. Die Kräfteverhältnisse im südchinesischen Meer tendieren zu einer vetofähigen Koalition gegen den Revisionismus Chinas, das somit wenig Aussichten hat, seine Ansprüche einseitig durchzusetzen, und in der Taiwan-Frage geht es nur um die Kodifizierung der jetzigen Verhaltensmuster. Historisches Vorbild für eine friedensgerichtete Großmachtkooperation könnte das Europäische Konzert des 19. Jahrhunderts sein, in dem die europäischen Mächte trotz fortbestehender Interessenunterschiede im »Friedensmanagement « zusammenarbeiteten, um die Wiederkehr des großen Krieges zu verhindern (Evera 2008). ${ }^{13}$

Die nuklearen Abrüstungsschritte müssten im Rahmen einer Transformationsstrategie so konzipiert sein, dass sie eine zunehmende Konzertierung der Sicherheit der Großmächte durch Vertrauensbildung unterstützen. Die folgende Agenda stellt denkbare Schritte und ihre vermuteten Wirkungen exemplarisch vor.

Ein Fissile-Material-Cut-off-Vertrag (FMCT) zielt auf ein weltweites Verbot der Spaltstoffproduktion für Waffenzwecke. Die Verhandlungen liegen gegenwärtig wegen der durch Pakistan verantworteten Blockade der Genfer Abrüstungskonferenz auf Eis, könnten aber theoretisch unter den übrigen Kernwaffenmächten in einem gesonderten Verhandlungsprozess in Angriff genommen werden. Ein FMCT hat aus einer Abrüstungsperspektive nicht nur den Vorteil, eine (wenngleich in einigen Fällen hohe) Obergrenze für den Ausbau der bestehenden Arsenale zu ziehen. Das fällige Verifikationssystem, dessen Verlässlichkeit und Präzision jenes des Nichtverbreitungsvertrages nicht unterschreiten dürfte, würde Transparenz über

13 Matthias Schulz (2009) räumt mit dem Irrtum auf, das Konzert sei lediglich ein konservatives Machterhaltungskartell gewesen und zeigt, wie es auch friedlichen Wandel unterstützt hat. 
zentrale Teile der nuklearen Komplexe in allen Kernwaffenstaaten schaffen. Diese geteilte Informationsbasis wird desto wichtiger werden, je mehr sich die Arsenale durch Reduktionsschritte verkleinern und je verlässlicher damit das Wissen über die Rekonstitutionsfähigkeiten der Partner werden muss (Schaper 2011). Sie bildet daher die Voraussetzung für die finalen Abrüstungsschritte und für das Verifikationssystem einer kernwaffenfreien Welt. Zugleich signalisiert sie Kernwaffenstaaten wie Nichtkernwaffenstaaten den ernsthaften Willen der Ersteren, sich auf eine solche Welt zuzubewegen und stiftet damit politisches Vertrauen.

Transparenz in der Nuklearpolitik bildet ein zentrales Kriterium für die Beziehungsgüte der Beteiligten. Das Lernen dieses Erfordernisses ist für Militärs und klassische Sicherheitspolitiker kontraintuitiv. Deckung und Überraschung sind wesentliche Pluspunkte in militärischen Dispositiven und vor allem für die schwächeren Parteien von disproportionaler Wichtigkeit (wie China nicht müde wird zu betonen). Beide Konzepte stehen im Gegensatz zu Transparenz. Es besteht daher ein beträchtliches Bereitschaftsgefälle unter den Kernwaffenstaaten, Transparenzmaßnahmen zu akzeptieren, das nicht vollständig entlang der Achse Demokratie/NichtDemokratie verläuft. Dies zeigt das Beispiel Indien, das sich mit Transparenz ausgesprochen schwer tut - wenngleich Autokratien natürlich ihre besondere Mühe haben, sich den einschlägigen Erfordernissen anzubequemen (Schaper/Müller 2009). Seit 2009 gibt es indes, fast unbemerkt von der Öffentlichkeit, regelmäßige hochrangige Treffen und von diesen eingesetzte Arbeitsgruppen auf Expertenebene, in denen sich die Kernwaffenstaaten bemühen, miteinander ins Gespräch über nukleare Fragen zu kommen. Erste Errungenschaft ist die Arbeit an einem Begriffsglossar, ein bescheidener, aber nicht zu unterschätzender Schritt in Richtung Transparenz (Kile 2012). Indien sollte so bald als möglich unbedingt einbezogen werden.

Diese Gespräche könnten in absehbarer Zeit auch ein »Deckelungsabkommen « in Betracht ziehen, in dem die kernwaffenbesitzenden Staaten eine Obergrenze für ihre Arsenale für einen bestimmten Zeitpunkt in der Zukunft festlegen. Diese Marge müsste nicht zwangsläufig einheitlich für alle Parteien sein - Großbritannien und Frankreich beispielsweise dürften keinen Bedarf nach einer Steigerung ihrer Bestände verspüren. Gleichwohl könnte eine solche Regelung - wie damals SALT I (Strategic Arms Limitation Talks) - für einige Teilnehmer (China, Indien?) Spielraum für einen gewissen Aufwuchs lassen. ${ }^{14}$ Die Parteien könnten ferner eine Selbstverpflichtung eingehen, künftig in weiteren Abrüstungsschritten auf eine gleiche Bestandszahl zu konvergieren. Ein solcher Deckelungsvertrag würde vor

14 So wie der angepasste KSE-Vertrag headroom zwischen den aktuellen und erlaubten Beständen für viele Vertragsparteien ließ. 
allem hinsichtlich der indischen und chinesischen Pläne Erwartungssicherheit schaffen, die heute nicht besteht. Dieser Sicherheitsgewinn würde nicht beeinträchtigt, wenn die Partner ihren Ratifikationsurkunden Konditionen für die dauerhafte Vertragstreue beifügen würden, etwa die Konditionierung auf eine Begrenzung der Raketenabwehr in Partnerstaaten, worauf Russland und China voraussichtlich beharren würden. Damit wären zumindest die Parameter der sicherheitspolitischen Debatte in den USA unmissverständlich klar, was den Rüstungskontrollbefürwortern im Vergleich zum Status Quo eher Vorteile verschaffen könnte.

Die Absenkung der Alarmstufe (De-alerting) der nuklearen Streitkräfte hat das Interesse der Four Horsemen auf sich gezogen. Hierzu hat die Nuclear Threat Initiative, eine Nichtregierungsorganisation, die als operativer Arm der Vier fungiert, eine Reihe konkreter Vorschläge entwickelt. Mit dem schrittweisen De-alerting dokumentieren die Kernwaffenstaaten, dass der Erstschlag kein Teil ihrer Nuklearstrategie ist und dass sie dergleichen auch nicht von ihren Partnern befürchten (Miller/Davis 2009). Damit ist der Kern des nuklearen Sicherheitsdilemmas betroffen. Die resultierende Vertrauensbildung ließe sich noch steigern, wenn in den Einsatzzentralen aller beteiligten Staaten Verbindungsoffiziere aller Kernwaffenstaaten mit störsicheren Kommunikationslinien zu ihren Hauptquartieren platziert würden.

Schließlich könnte sich die Kooperation auch auf die Doktrinen beziehen. Der »Nichtersteinsatz« (Feiveson/Hoogendorn 2003) ist die logische Grundlage einer vollständigen nuklearen Abrüstung: Wenn man Kernwaffen nur noch zur Vergeltung gegen einen Kernwaffeneinsatz zu benötigen glaubt, steht ihrer simultanen Abschaffung in allen Kernwaffenstaaten nichts entgegen. Bekanntlich ist das gegenwärtige Set von Doktrinen ein bunter Strauß; kaum zwei sind deckungsgleich. Während die russische Doktrin zunächst auf eine »Konventionalisierung « der Kernwaffen zuzulaufen schien, ist dieser Trend in der letzten Version der Nationalen Sicherheitsstrategie nicht vertieft worden; der Ersteinsatz wurde auf Lagen beschränkt, in denen das nationale Überleben auf dem Spiel steht. Die amerikanische Doktrin hat sich im gleichen Zeitraum in Richtung Nichtersteinsatz bewegt; noch bedeutsamer ist die Willensbekundung der USA, an den Voraussetzungen für eine Nichtersteinsatzdoktrin arbeiten zu wollen (Müller et al. 2010). In einer Debatte über Doktrinen müssten die Nuklearmächte klären, welche Bedrohungslagen für sie den Rückgriff auf den ersten Einsatz von Kernwaffen rechtfertigen und was die Partner zu tun hätten, um diese Bedrohungen und damit die Hindernisse für das gemeinsame Bekenntnis zum Nichtersteinsatz zu beseitigen. Eine Doktrin des Nichtersteinsatzes macht aber hohe Sprengkopfzahlen und ein ausgefächertes 
Arsenal nichtstrategischer Kernwaffen überflüssig; das Festhalten daran würde kritische Fragen an die Partner aufwerfen.

\section{Paradigmenwechsel: Überwindung der Abschreckungsdogmatik}

Das Dilemma der nuklearen Abrüstungspolitik ist also, transitorisch auf Abschreckung als Sicherheitsinstrument zu fußen, von dieser aber in vielfältiger Weise behindert zu werden. Der Übergang ist also so zu gestalten, dass die negativen Wirkungen fortgesetzter Abschreckung abgebaut, neutralisiert und schließlich überwunden werden.

\subsection{Von der Abschreckung zur kooperativen Sicherheitspolitik}

Die vorangegangenen Ausführungen beziehen sich auf einen Zukunftszeitraum von zehn bis fünfzehn Jahren. Während dieses Zeitraums bleibt die nukleare Abschreckung zwischen den Großmächten die Rückfallposition. Die Möglichkeit, auf diese Option für die eigene Sicherheitspolitik zurückzugreifen, falls der Abrüstungsprozess nicht den erhofften Sicherheitsgewinn erbringt, ist anfänglich Teil der Motivation, es überhaupt zu versuchen. Zugleich zielen die vorgeschlagenen Schritte darauf ab, diese Rückfallposition immer weiter in den Hintergrund zu schieben und Stück für Stück die Gründe auszuräumen, die in den Augen der Sicherheitspolitiker nukleare Abschreckung zwingend machen, sodass sie in einem weiteren Horizont überflüssig werden könnte.

Bei günstigem Verlauf könnten sich in diesem Zeitraum die relativen Bewertungen verschieben, die nukleare Abschreckung einerseits, kooperative Sicherheit andererseits als sicherheitspolitische Instrumente erfahren. Gegenwärtig ist nukleare Abschreckung das Standbein, kooperative Sicherheit das Spielbein; Abschreckung stellt die Normalität, kooperative Sicherheit die Anomalie nationaler Sicherheitspolitiken dar. Der Vertrauensbildungsprozess ist darauf angelegt, dieses Verhältnis umzukehren. Nur so lässt sich das »mentale Sprungbrett« konstruieren, von dem aus der Sprung in die kernwaffenfreie Welt denkbar wird.

Aber selbst in der Wahrnehmung als Anomalie bildet die nukleare Abschreckung einen Störfaktor für die nukleare Abrüstung: in mentaler Perspektive, weil das nukleare Sicherheitsdilemma, wenn auch in abgeschwächter Form, erhalten bleibt Abschreckungspolitik bedeutet stets, dass der Nuklearkrieg zwar unwahrscheinlicher wird, aber ein Stück Restwahrscheinlichkeit besteht, dass er stattfinden könnte. Damit lebt ein Residuum der Unterstellung fort, einer der Partner könne einen solchen Krieg provozieren oder starten. Feindbilder wären zwar im Vergleich zur Gegenwart fast zur Unkenntlichkeit verblasst, aber eben nur fast. Sie werden besonders 
schwach sein, wenn sich alle Beteiligten glaubwürdig zum Nichtersteinsatz bekennen. Insofern zeigt sich, dass die »symbolische Politik« der Veränderung von Doktrinen ihre Rolle in der Transformation zu spielen hat.

\subsection{Neutralisierung der Kernwaffenlobby}

Politisch-institutionell wirkt der Fortbestand der Kernwaffenkomplexe schwer. Dort lebt das bürokratische Eigeninteresse an institutioneller Unsterblichkeit, also der Fortsetzung ihres Kerngeschäfts, fort. Dort wird der fruchtbare Boden für kontinuierliche Lobbyarbeit gegen die nukleare Abrüstung weiterleben. Der Einfluss dieser Formationen wird sich zwar abschwächen, aber immer noch ein Hindernis in der Endphase nuklearer Abrüstung darstellen. Wie die amerikanische Nuklearbürokratie 1994 gegen einen widerstrebenden Verteidigungsminister eine abrüstungsfreundlichere Nukleardoktrin verhindert hat, stellt ein Lehrstück dar (Nolan 1999).

Diese Konstellation ist nicht zu vermeiden, aber die Positionierung von Gegengewichten ist möglich, um den Einfluss der Kernwaffenlobby zu schwächen. Hierfür ist der von der Schweiz, Österreich und anderen Nichtkernwaffenstaaten auf der NVV-Überprüfungskonferenz von 2010 eingeschlagene Weg richtungweisend: Anstrengungen zur weiteren Delegitimierung der Kernwaffen durch das Bewusstmachen ihres inhumanen Charakters zu unternehmen. ${ }^{15}$ Es geht um eine schrittweise Ächtung nach dem Modell der chemischen und biologischen Waffen, die in der Zielsetzung einer Welt ohne Kernwaffen ohnedies angelegt ist. Je stärker sich diese Norm verankert, desto schwerer wird der Stand der Kernwaffenlobby werden und desto nachhaltiger wird die Position derjenigen Politiker gestärkt, die auf Abrüstung hinarbeiten.

Die Kernwaffenkomplexe sollten zunehmend Abrüstungsaufgaben durchführen und dafür bezahlt werden. Das schafft gegengewichtige bürokratische Teilinteressen, sodass eine einheitliche Linie, den Abrüstungsprozess zu sabotieren, schwer durchzuhalten sein wird. Modelle sind die internationalen Wissenschaftszentren, die nach dem Zerfall der Sowjetunion in Moskau und Kiew eingerichtet wurden, um von der Arbeitslosigkeit betroffene Waffenexperten zu beschäftigen, oder die Beauftragung des britischen Kernwaffenforschungszentrum Aldermaston mit experimentellen Arbeiten zur Verifikation einer kernwaffenfreien Welt (Milne/Wilson 1998).

15 Vgl. hierzu die im Auftrag der schweizerischen Regierung erstellte Studie von Berry et al. (2010). 


\subsection{Kollektive statt nationale Abschreckung gegen Regelbrüche}

In der Diskussion der Abschreckung während der langen Übergangsphase fehlt noch der Aspekt der kleineren Staaten im Besitz von Kernwaffen oder anderen »Massenvernichtungswaffen" (Hagerty 2012; Ogilvie-White 2012). Die Doktrin des Nichtersteinsatzes verlangt ja ohnedies die Bekämpfung von biologischen und chemischen Waffen mit konventionellen Mitteln. Im Verhältnis der Kernwaffenstaaten zu nuklear ambitionierten Staaten ist zudem eine Dynamik in Rechnung zu stellen, die abrüstungskritische Analysen vernachlässigen: die Veränderung der Großmachtinteressen durch den Abrüstungsprozess.

Je weiter sich die Arsenale der Großmächte der Null nähern, desto bedeutsamer wirken aus Sicherheits- und Statusgründen die Bestände Dritter. Die Interessen an der Nichtverbreitung und am »Rollback« nuklearer Aufrüstungsprozesse außerhalb der Großmachtkonkurrenz werden konvergieren. Wo diese Interessen heute unter geopolitischen und geostrategischen Perspektiven abgewogen werden, wird sich das Gewicht zugunsten des gemeinsamen Interesses verlagern, Drittstaaten keinen Statusgewinn gegenüber den heutigen Kernwaffenmächten zuzumessen. Der Druck auf Dritte, sich dem Abrüstungsprozess zu unterwerfen oder Kernwaffenaktivitäten einzustellen, wird steigen. Das betrifft die Beziehungen China/Nordkorea, China/ Pakistan, China/Russland/Iran, aber auch USA/Israel. Die resultierende diplomatische Zusammenarbeit zwischen den Großmächten, um die renitenten Schützlinge zum Einlenken zu bewegen, könnte sich zu einem besonders wirksamen Feld kooperativer Sicherheit entwickeln.

Sicherheit in einer kernwaffenfreien Welt verlangt nach einem neuen Enforcement-Verfahren. Die immer wieder auftretenden Lähmungen im Sicherheitsratbieten zu wenig Verlässlichkeit bei Vertragsbrüchen. Wenn die für die Überwachung der Kernwaffenfreiheit zuständige Verifikationsorganisation »Gefahr im Verzug« durch ein Kernwaffenprogramm feststellt, sollte dies zu einer Krisensitzung des Sicherheitsrats sowieeinerSondersitzungderVollversammlung führen, dieimFalleeinerBlockade des Ersteren unter der Uniting-for-Peace-Prozedur ein Eingreifen ihrer Mitglieder legitimieren (wenn auch nicht mandatieren) könnte. Zugleich könnte diese Feststellung von »Gefahr im Verzug « aufgrund einer Klausel in der Kernwaffenkonvention, welche die kernwaffenfreie Welt konstituiert, die Mitgliedsstaaten zur SelbstverteidigungnachArt. 51 derChartaermächtigen.EsgäbealsodreilegaleHandlungsstränge, die zur Aktion gegen den Regelbrecher führen könnten. Der (kollektive) Abschreckungswert dieses Arrangements wäre gegenüber dem Status Quo höher.

Die Stärkung kooperativer Sicherheit dürfte auch das wirksamste Abschreckungsinstrument gegenüber Besitzern biologischer und chemischer Waffen wer- 
den. Abschreckung wandelt sich vom Normalfall nationaler Sicherheitspolitiken im Zentrum des internationalen Geschehens zu einem Ausnahmefall kollektiver Sicherheitspolitik an der Peripherie des internationalen Geschehens (Müller 2010).

\subsection{Virtuelle Abschreckung: Transitionsphase oder Sackgasse?}

Die Gretchenfrage einer Transformationsstrategie lautet: Was wird aus der Abschreckung in einer Welt ohne Kernwaffen? Die prominenteste Antwort lautet: Sie bleibt in virtueller Form erhalten (Drell/Goodby 2010). Anstelle der physischen Kernwaffendispositive stützt sie sich auf virtuelle Arsenale, die aus der Kombination von residualen technisch-materiellen Voraussetzungen und einer Rekonstitutionsfähigkeit bestehen (Mazarr 1997). Nun werden »virtuelle Arsenale« in einer Übergangsphase, in der die letzten Waffen und die einschlägigen Anlagen demontiert und die Spaltmaterialien dem zivilen Brennstoffkreislauf zugeführt oder transmutiert werden, unvermeidlich sein. Darin aber das Endstadium nuklearer Abrüstung zu sehen, ist aus mehreren Gründen unhaltbar.

Von Kernwaffenkomplexen geht ein beständiger politischer Druck zur Erhaltung oder Rekonstitution der Kernwaffenarsenale aus (siehe oben). Eine Suborganisation im Staatsapparat, deren einzige Aufgabe es ist, für den Ernstfall den Bruch der Norm der Kernwaffenfreiheit vorzubereiten, verlängert diesen Druck in die unbestimmte Zukunft und unterläuft das wichtigste Gegengewicht, die moralische und rechtliche Ächtung der Kernwaffen. Damit wird das nukleare Sicherheitsdilemma ad calendas graecas gedehnt. Jede Großmacht wüsste von der anderen, dass sie einen Apparat mit dem Potenzial schneller Rekonstitution vorhält. Eine Welt, in der eine Reihe von Staaten - nicht nur die heutigen Kernwaffenbesitzer - ein paar Schraubendrehungen von der Bombe entfernt ist, ist extrem instabil. Thomas Schelling (2009) hat eine so konfiguriertenicht-nukleare Welt gefährlicherals diejetzige genannt, weil eineKrise zum Wettlauf um die Rekonstituierung der Kernwaffenfähigkeit führen würde.

Tatsächlich stellt das Zustandekommen eines Abschreckungssystems, das sich dauerhaft auf virtuelle Arsenale stützt, eigentlich eine logische Unmöglichkeit dar: Es ist nicht vorstellbar, dass Regierungen, die glauben, ihre Sicherheit bedürfe der Fähigkeit, Kernwaffen schnell zu rekonstituieren, den letzten Schritt der kompletten Demontage aller Kernwaffen und der Einlagerung ihrer Bestandteile tun würden. Der Vergleich der Sicherheitsgewährleistung eines solchen Dispositivs mit einem physischen Arsenal von wenigen hundert Kernwaffen fällt - wenn man an der Abschreckung festhalten will - aufgrund der besseren Stabilitätswerte zugunsten des Letzteren aus. Die Realisierbarkeit einer auf virtuelle Abschreckung gegründeten 
Welt scheitert an der rationalen Präferenzordnung, die das Abschreckungsparadigma zugunsten von physischen und gegen virtuelle Arsenale ergibt (Müller 2013).

Das Ziel einer kernwaffenfreien Welt verlangt es daher, auch die Mentalitäten von der Idee der nuklearen Abschreckung zu befreien. »Kernwaffenfrei« ist keine ausschließlich physikalisch-technische Befindlichkeit, sondern schließt kulturelle und psychologische Komponenten ein (Herz 2013). Der beste Vergleich ist das Verschwinden des Denkens an die Kriegsmöglichkeit zwischen den Staaten der Kern-EU: Nur so ist eine kernwaffenfreie Welt langfristig vorstellbar, und nur so kann nukleare Abrüstung ihre strategische Rolle bei der »Entfeindung« der Großmächtebeziehungen bis zum Ende spielen.

\section{Schlussfolgerung: Der Weg ist das Ziel, aber das Ziel bestimmt den Weg}

»Das Endziel ist nichts, die Bewegung alles« charakterisierte der sozialdemokratische Reformer Eduard Bernstein die Arbeit an der sozialen Gerechtigkeit, den konfuzianischen Gedanken »der Weg ist das Ziel« politisch wendend. Ebenso sollte man auch das Projekt der nuklearen Abrüstung im Rahmen einer weltpolitischen Transformationsstrategie betrachten. Der Ansatz will globalen Schaden vermeiden und globale Risiken mindern. Auf den nuklearen Abrüstungsprozess angewandt, bedeutet das Bernstein-Prinzip, dass die einzelnen Maßnahmen, von denen in diesem Papier eine Auswahl diskutiert wurde, sowie die Arbeit an den politischen Beziehungen zwischen den Großmächten, ohne die diese Maßnahmen nicht zu Stande kommen und die umgekehrt von ihnen ermöglicht und unterstützt werden, die Welt bereits schrittweise sicherer machen. Selbst wenn das Endziel nicht erreichbar sein sollte - und wer weiß das heute? - wird sich die Arbeit gelohnt haben: Es bleibt ein höheres Maß an Reversibilität bestehen als in einer vollständig nuklear abgerüsteten Welt, aber der Sicherheitsgewinn könnte ausreichen, um ein dauerhaftes Management der Großmächtebeziehungen zu etablieren.

Damit diese Dialektik funktioniert, muss man gleichwohl das Ziel vor Augen haben, und man muss ihm die richtige Form geben. Um dazu beizutragen, wurden in diesem Aufsatz zwei Auffassungen kritisiert: Zum einen wurde die Vorstellung zurückgewiesen, dass die nukleare Abrüstung ein von der Politik abgekoppelter, selbstlaufender Prozess sein könnte. Dies unterstellen nicht nur manche Abrüster, sondern paradoxerweise auch ihre härtesten Gegner, die in ihren Argumentationen zumeist die heutigen Parameter der Weltpolitik voraussetzen und dann zeigen, dass nukleare Abrüstung unter diesen Voraussetzungen unmöglich sei. ${ }^{16}$ Dem hält mein

Rühle (2009) ist das deutsche Paradebeispiel für diesen Irrtum. 
Konzept entgegen, dass sich verändernde Praktiken sowohl die Verhältnisse verändern, in denen sie stattfinden, als auch die Akteure, die sie anwenden (Lebow 2003: 323-330). Zum anderen wurde die umgekehrte - optimistische - Variante als logische und praktische Unmöglichkeit dekonstruiert, dass die nukleare Abschreckung in ihrer virtuellen Variante die vollständige Abrüstung erst möglich mache. Denn Abrüstung ist kein rein physikalisch-technischer, sondern in viel höherem Maße ein mentaler, kultureller und psychologischer Prozess, ohne den physikalischtechnische Veränderungen letztlich bedeutungslos bleiben.

Eine solche Änderung findet nur statt, wenn ein Akteur mit Führungsqualitäten als Katalysator für neue Praktiken wirkt. Die Obama-Administration hat zu Beginn Anstrengungen unternommen, in eine solche Rolle hineinzuwachsen. Sie hat die multilaterale Rüstungskontrolle wiederbelebt und unilaterale, exklusive Initiativen der Bush-Administration zum Teil multilateralisiert (Meier 2013: 55-58). Dieser Politikwandel hat positive Ergebnisse erbracht, aber noch keinen Durchbruch. Die USA als der militärisch stärkste Akteur auf der Weltbühne haben die beste Chance, die Weichen zu stellen, wie Gorbatschow dies nach 1985 tat. Inwieweit die innenpolitische Konstellation der USA dies zulässt, ist eine hochkontingente Frage. In den wahrgenommenen Interessenlagen und den sicherheitspolitischen Kulturen der übrigen, in die zentrale Konfliktkonstellation verwickelten Großmächte gibt es Momente, die dem Abrüstungsprozess entgegenkommen und andere, die ihm zuwiderlaufen; der Prozess und sein Ausgang sind insoweit nicht determiniert. ${ }^{17}$ Andere Akteure können Einfluss zugunsten der Abrüstung ausüben. Die Initiative einiger Staaten, die humanitären Kosten des Kernwaffenkrieges neu zu thematisieren, geht in diese Richtung, ebenso die transnationalen zivilgesellschaftlichen Aktivitäten von Organisationen wie Global Zero, ICAN (International Campaign to Abolish Nuclear Weapons) oder IPPNW (International Physicians for the Prevention of Nuclear War).

$\mathrm{Ob}$ die nukleare Abrüstung geschehen wird oder nicht, vermag heute niemand zu prognostizieren. Prophezeiung ist nicht das Geschäft der sicherheitspolitischen Analyse und der Friedensforschung. Vielmehr besteht die Aufgabe bei diesem Thema in der Ausmessung politischer Möglichkeitsräume, es geht um klare Begriffe und um kohärente Konzepte. Eine nuklearwaffenfreie Welt ist unter bestimmten Voraussetzungen möglich, und der Weg dorthin auch. Ob er eingeschlagen wird, ist eine Frage des gesellschaftlichen Drucks, der politischen Praxis und der politischen Weitsicht und Weisheit.

17 Es fehlt der Raum, um die ambivalenten Interessenlagen der Großmächte und ihre möglichen Effekte auf nukleare Abrüstung detailliert zu behandeln. Gute Hinweise zu einer solchen Analyse finden sich in den einschlägigen Kapiteln in Ogilvie-White/Santoro (2012). 


\section{Literatur}

Adler, Emanuel/Barnett, Michael (Hrsg.) 1998: Security Communities, Cambridge. Angell, Norman 1910: Die große Täuschung. Eine Studie über das Verhältnis zwischen Militärmacht und Wohlstand der Völker, Leipzig.

Archibugi, Daniele 2008: The Global Commonwealth of Citizens: Toward Cosmopolitan Democracy, Princeton, NJ.

Beck, Ulrich 2004: Der kosmopolitische Blick oder: Krieg ist Frieden, Frankfurt a. M.

Beckley, Michael 2011: China's Century? Why America's Edge Will Endure, in: International Security 36: 3, 41-78.

Berry, Ken/Lewis, Patricia/Pélopidas, Benoît/Sokov, Nikolai/Wilson,Ward 2010:

Delegitimizing Nuclear Weapons: Examining the Validity of Nuclear Deterrence, Monterey, CA.

Bloch, Jean de [1902] 1972: The Future of War in its Technical, Economic and Political Relations, New York, NY.

Boli, John/Thomas, George (Hrsg.) 1999: Constructing World Culture: International Nongovernmental Organizations Since 1875, Stanford, CA.

Booth, Ken (Hrsg.) 2005: Critical Security Studies and World Politics, Boulder, CO.

Booth, Ken/Wheeler, Nicholas J. 2008: The Security Dilemma: Fear, Cooperation and Trust in World Politics, Houndmills.

Brandt, Willy 1968: Friedenspolitik in Europa, Frankfurt a. M.

Causevic, Amar 2012: A Thirsty Dragon: Rising Chinese Crude Oil Demand and Prospects for Multilateral Energy Security Cooperation (PRIF Report No. 116), Frankfurt a. M.

Cimbala, Stephen J. 2013: Arms for Uncertainty: Nuclear Weapons in US and Russian Security Policy, Farnham.

Clausewitz, Carl von 1980: Vom Kriege. Ungekürzter Text nach der Erstauflage, Frankfurt a. M.

Czempiel, Ernst-Otto 1979: Amerikanische Außenpolitik. Gesellschaftliche Anforderungen und politische Entscheidungen, Stuttgart.

Czempiel, Ernst-Otto 1989: Machtprobe. Die USA und die Sowjetunion in den 80er Jahren, München.

Delpech, Thérèse 2012: Nuclear Deterrence in the 21 ${ }^{\text {st }}$ Century: Lessons from the Cold War for a New Era of Strategic Piracy, Santa Monica, CA.

Dessler, David 1989: What's at Stake in the Agent-Structure Debate, in: International Organization 43: 3, 441-473. 
Drell, Sidney D./Goodby, James E. 2010: A World without Nuclear Weapons: EndState Issues, in: http://npsglobal.org/eng/images/stories/nukefree/4\%20sidney_ drell_james_goodby_a_world_without_nuclear_weapons_1.pdf; 22.12.2012.

English, Robert D. 2000: Russia and the Idea of the West: Gorbachev, Intellectuals, and the End of the Cold War, New York, NY.

Evangelista, Matthew 2004: Turning Points in Arms Control, in: Herrmann, Richard K./Lebow, Richard Ned (Hrsg.): Ending the Cold War: Interpretations, Causation, and the Study of International Relations, Houndmills, 83-106.

Evera, Stephen van 2008: A Farewell to Geopolitics, in: Leffler, Melvyn P./Legro, Jeffrey W. (Hrsg.): To Lead the World: American Strategy after the Bush Doctrine, Oxford, 10-35.

Feiveson, Harold A./Hogendoorn, Ernst Jan 2003: No First Use of Nuclear Weapons, in: The Nonproliferation Review 10: 2, 1-9.

Fingar, Thomas 2011: Worrying about Washington: China's Views on the US Nuclear Posture, in: The Nonproliferation Review 18: 1, 51-68.

Foot, Rosemary/Walter, Andrew 2011: China, the United States, and Global Order, Cambridge.

Giddens, Anthony 1984: The Constitution of Society: Outline of the Theory of Structuration, Cambridge.

Gilboy, George J./Heginbotham, Eric 2012: Chinese and Indian Strategic Behavior: Growing Power and Alarm, Cambridge.

Goldstein, Avery 2013: First Things First: The Pressing Danger of Crisis Instability in U.S.-China Relations, in: International Security 37: 4, 49-89.

Gray, Colin S. 1992: House of Cards: Why Arms Control Must Fail, Ithaca, NY.

Grieco, Joseph M. 1990: Cooperation among Nations: Europe, America, and Nontariff Barriers to Trade, Ithaca, NY.

Hagerty, Devin T. 2012: The Nuclear Holdouts: India, Israel and Pakistan, in: Ogilvie-White, Tanya/Santoro, David (Hrsg.): Slaying the Nuclear Dragon: Disarmament Dynamics in the Twenty-First Century, Athens, GA, 219-248.

Herz, Monica 2013: The Cultural Changes Needed for the Abolition of Nuclear Weapons, in: Atwood, David/Munro, Emily J. (Hrsg.): Security in a World without Nuclear Weapons: Visions and Challenges, Genf, 39-48.

Homolar, Alexandra 2012: US Security Policy: Origins, Concepts, and Contemporary Challenges, London.

ICNND - International Commission on Nuclear Non-Proliferation and Disarmament 2009: Eliminating Nuclear Threats: A Practical Agenda for Global Policymakers, Canberra. 
Jervis, Robert 1976: Perceptions and Misperception in International Politics, Princeton, NJ.

Kagan, Robert 2008: The Return of History and the End of Dreams, New York, NY. Kile, Shannon N. 2012: International Cooperation on Non-proliferation, Arms Control and Nuclear Security, in: Stockholm International Peace Research Institute (Hrsg.): SIPRI Yearbook 2012, Oxford, 387-390.

Kile, Shannon N./Fedchenko, Vitaly/Glaser, Alexander/Kristensen, Hans M./Mia, Zian/Schell, Philip 2012: World Nuclear Forces, in: Stockholm International Peace Research Institute (Hrsg.): SIPRI Yearbook 2012, Oxford, 319-359.

Lebow, Richard Ned 2003: The Tragic Vision of Politics: Ethics, Interests and Orders, Cambridge.

Lebow, Richard Ned 2008: A Cultural Theory of International Relations, Cambridge.

Lebow, Richard Ned 2010 a: Forbidden Fruit: Counterfactuals and International Relations, Princeton, NJ.

Lebow, Richard Ned 2010 b: Why Nations Fight: Past and Future Motives for War, Cambridge.

Lebow, Richard Ned/Stein, Janice Gross 1994: We All Lost the Cold War, Princeton, NJ.

Lemke, Douglas/Tammen, Ronald L. 2006: Power Transition Theory and the Rise of China, in: International Interactions 22: 2, 143-164.

Lewis, Jeffrey 2007: Minimum Means of Reprisal: China's Search for Security in the Nuclear Age, Cambridge, MA.

Mazarr, Michael J. (Hrsg.) 1997: Nuclear Weapons in a Transformed World: The Challenge of Virtual Arsenals, Houndmills.

Meier, Oliver 2013: Non-cooperative Arms Control, in: Meier, Oliver/Daase, Christopher (Hrsg.): Arms Control in the $21^{\text {st }}$ Century: Between Coercion and Cooperation, London, 39-66.

Mercer, Jonathan 2010: Emotional Beliefs, in: International Organization 64: 1, $1-31$.

Mercer, Jonathan 2013: Emotion and Strategy in the Korean War, in: International Organization 67: 2, 221-252.

Meyer, John W. 2010: World Society: The Writings of John W. Meyer, New York, NY.

Miller, Tim/Davis, Rebecca 2009: De-alerting Nuclear Forces, Colorado Springs, $\mathrm{CO}$.

Milne, Tom/Wilson, Henrietta 1998: Aldermaston and Nuclear Disarmament, in: Bulletin of the Atomic Scientists 54: 6, 24-25. 
Mohan, Raja C. 2003: Crossing the Rubicon: The Shaping of India's New Foreign Policy, London.

Müller, Erwin/Neuneck, Götz 1992: Rüstungsmodernisierung und Rüstungskontrolle. Neue Technologien, Rüstungsdynamik und Stabilität, Baden-Baden.

Müller, Harald 2006: Indien. Wie uns der rasante Aufstieg herausfordert, Frankfurt a. M.

Müller, Harald 2010: Enforcement of the Rules in a Nuclear Weapon-Free World, in: Hinderstein, Corey (Hrsg.): Cultivating Confidence: Verification, Monitoring, and Enforcement for a World Free of Nuclear Weapons, Washington, D.C., 33-66.

Müller, Harald 2012 a: Über allen Gipfeln ist Ruh. Zum Verhältnis von Friedensforschung und IB, in: Zeitschrift für Internationale Beziehungen 19: 1, 155-169. Müller, Harald 2012 b: The NPT Process and Strengthening the Treaty: Disarmament (EU Non-Proliferation Consortium Non-Proliferation Papers No. 10), Stockholm.

Müller, Harald 2013: Icons Off the Mark: Waltz and Schelling on a Perpetual Brave Nuclear World, in: Nonproliferation Review 20: 3, 545-565.

Müller, Harald/Fey, Marco/Franceschini, Giorgio/Schmidt, Hans-Joachim 2010:

Auf dem Weg zu Global Zero? Die neue amerikanische Nuklearpolitik zwischen Anspruch und Wirklichkeit (HSFK-Report Nr. 4), Frankfurt a. M.

Müller, Harald/Schaper, Annette 2009: Transparenz für die kernwaffenfreie Welt.

Konzepte für ein Kernwaffen- und Spaltmaterialregister (HSFK-Report Nr. 10), Frankfurt a. M.

Müller, Harald/Schörnig, Niklas 2006: Rüstungsdynamik und Rüstungskontrolle.

Eine exemplarische Einführung in die Internationalen Beziehungen, Baden-Baden.

Mueller, John E. 1989: Retreat from Doomsday: The Obsolescence of Major War, New York, NY.

Neuneck, Götz/Rothkirch, André 2006: Weltraumbewaffnung und Optionen für präventive Rüstungskontrolle, Osnabrück.

Nolan, Janne E. 1999: An Elusive Consensus: Nuclear Weapons and American Security after the Cold War, Washington, D.C.

Nye, Joseph S. 2002: The Paradox of American Power: Why the World's Only Superpower Can't Go it Alone, New York, NY.

Ogilvie-White, Tanya/Santoro, David 2012 (Hrsg.): Slaying the Nuclear Dragon:

Disarmament Dynamics in the Twenty-First Century, Athens, GA.

Ogilvie-White, Tanya 2012: The Defiant States: North Korea and Iran, in: OgilvieWhite/Santoro 2012, 249-278. 
Osgood, Charles E. 1970: An Alternative to War or Surrender, Urbana, IL.

Perkovich, George/Acton, James M. 2008: Abolishing Nuclear Weapons, Abingdon.

Pfaltzgraff, Robert Jr. 2009: China-U.S. Strategic Stability, in: http://www.ifpa.org/ pdf/Pfaltzgraff_China-US.Strategic.Stability_Carnegie.Seminar_6.April.2009. pdf; 22.12.2012.

Pinker, Steven 2011: The Better Angels of Our Nature: Why Violence has Declined, New York, NY.

Rafii, Rahi 2009: Asiatische Rivalen: China versus Indien, in: Blätter für deutsche und internationale Politik 2/2009, 22-25.

Rauch, Carsten 2014: On Peaceful Power Transition - Die Machtübergangstheorie, das Konzept des friedlichen Machtübergangs und der weltpolitische Aufstieg Indiens, Baden-Baden.

Rosenau, James N. 1990: Turbulence in World Politics: A Theory of Change and Continuity, New York, NY.

Rühle, Michael 2009: Gute und schlechte Atombomben: Berlin muss die nukleare Realität mitgestalten, Hamburg.

Schaper, Annette 2011: A Treaty on Fissile Material: Just Cutoff or More? (PRIF Report No. 109), Frankfurt a. M.

Schulz, Matthias 2009: Normen und Praxis. Das Europäische Konzert der Großmächte als Sicherheitsrat, 1815-1860, München.

Schelling, Thomas C. 2009: A World Without Nuclear Weapons?, in: Daedalus 138: 4, 124-129.

Senghaas, Dieter (Hrsg.) 1970: Zur Pathologie des Rüstungswettlaufs. Beiträge zur Friedens- und Konfliktforschung, Freiburg i. Br.

Senghaas, Dieter 1972: Rüstung und Militarismus, Frankfurt a. M.

Shultz, George/Perry, William/Kissinger, Henry/Nunn, Sam 2007: A World Free of Nuclear Weapons (The Wall Street Journal, 04.01.2007), in: http://online. wsj.com/news/articles/SB116787515251566636; 09.03.2014.

Sicherheitsrat der Russischen Föderation 2009: Die Strategie der nationalen Sicherheit der Russischen Föderation bis zum Jahr 2020, in: http://www.scrf.gov.ru/ documents/99.html; 19.04.2010.

Sil, Rudra/Katzenstein, Peter J. 2011: Beyond Paradigms: Analytic Eclecticism in the Study of World Politics, Basingstoke.

Takahashi, Sugio 2012: Ballistic Missile Defense in Japan: Deterrence and Military Transformation (IFRI Proliferation Papers No. 44), Paris. 
Tammen, Ronald L./Kugler, Jacek/Lemke, Douglas/Alsharabati, Carole/Efird, Brian/Organiski, A. F. K. 2000: Power Transitions: Strategies for the $21^{\text {st }}$ Century, New York, NY.

Thränert, Oliver 1986: Rüstungssteuerung und Gradualismus. Möglichkeiten und Grenzen einer alternativen Sicherheitspolitik, München.

Wendt, Alexander 1987: The Agent-Structure Problem in International Relations Theory, in: International Organization 41: 3, 335-370.

Wendt, Alexander 1999: Social Theory of International Politics, Cambridge.

Yao, Yunzhu 2010: China's Perspective on Nuclear Deterrence, in: Air and Space Power Journal 24: 1, 27-30.

\section{Der Autor}

Prof. Dr. Harald Müller ist geschäftsführendes Vorstandsmitglied der Hessischen Stiftung Friedens- und Konfliktforschung (HSFK) und Professor für Internationale Beziehungen und Friedensforschung an der Goethe-Universität Frankfurt a. M.

E-Mail: mueller@hsfk.de 